\title{
Periprosthetic Joint Infection Caused by Mycoplasma hominis, Diagnosed Using Metagenomic Sequencing
}

\author{
Haiying Wang \\ Dongliang Ren \\ Hui Li \\ Shunyi Wang
}

Department of Orthopaedic Surgery, Baoding No.I Central Hospital, Baoding, 071000, People's Republic of China
Correspondence: Shunyi Wang Department of Orthopaedic Surgery, Baoding No.I Central Hospital, 320

Changcheng North Street, Baoding, Hebei, 07 1000, People's Republic of China Tel +8613021878068

Fax +863125975050

Email hbtswhyl@I63.com

\begin{abstract}
To our knowledge, the periprosthetic joint infection (PJI) caused by Mycoplasma hominis is a rare postoperative complication after total knee arthroplasty (TKA). Just a few cases associated with Mycoplasma hominis infection after TKA were reported all over the world currently. In view of the difficulty involved in isolating this microorganism, Mycoplasma hominis infection may be under-recognized and should be considered in culture-negative cases where the microbial etiology is not easily identified. Metagenomic sequencing is a new tool to identify pathogens undetected by conventional methods. In this report, we present a case where metagenomic sequencing was used to identify Mycoplasma hominis as a novel PJI pathogen after TKA in our hospital.
\end{abstract}

Keywords: knee, infection, Mycoplasma hominis, metagenomic sequencing

\section{Introduction}

Total knee arthroplasty (TKA) is a common and effective orthopedic procedure to treat severe osteoarthritis and rheumatoid arthritis. ${ }^{1}$ Periprosthetic joint infection(PJI) proves to be a serious complication following TKA. ${ }^{2}$ Although the overall incidence of PJI is low at $0.5-2.0 \%$ after primary TKA, it still brings a large burden on patients' health and finance. ${ }^{3,4}$ Mycoplasma hominis which exists mainly in the urinary tract and genital can also cause inflammation., 5he Mycoplasma hominis infection is a rare postoperative complication after TKA. Given the difficulty of isolating this microbe, Mycoplasma hominis PJI may be underestimated, making clinical treatment difficult. In this article, we have introduced metagenomic sequencing to identify this pathogen undetected by conventional methods. This report was approved by the Ethics Committee of Baoding No.1 Central Hospital and written informed consent has been provided by the patient to have the case details and any accompanying images published.

\section{Case Report}

A 54-year-old male patient was admitted to the orthopedic department of our hospital in November 2020 with bilateral knee osteoarthritis. The patient developed pain in both knees without obvious cause since 10 years ago. In addition, symptoms and joint function worsened despite conservative treatments of physical therapy and taking medicines. Over the past month, due to the severe knee pain which cannot be relieved by medication, the patient came to our hospital for further treatment. The patient was healthy without hypertension, coronary heart disease or diabetes. Both $\mathrm{C}$ reactive protein (CRP) and erythrocyte sedimentation rate (ESR) were normal 
before operation. Other laboratory results were all negative at admission. Then the patient accepted TKA on both sides under general anesthesia. The drainage tube was not placed routinely in our hospital after TKA surgery. The patient took cefamandole to prevent infection for about 72 hours postoperatively.

Due to postoperative pain and poor diet, hemoglobin dropped to $58.0 \mathrm{~g} / \mathrm{l}$ on the third day after surgery. So we transfused 4 units of red blood cells and $400 \mathrm{~mL}$ of plasma to improve anemia. Two days later, the patient experienced increasing pain on both knees and swelling on the left knee. The body temperature increased to $38.0^{\circ} \mathrm{C}$ without effusion of the two knees. The results of blood test suggested that CRP concentration increased to $78.7 \mathrm{mg} / \mathrm{l}$ and ESR to $121 \mathrm{~mm} /$ hour while hemameba did not change significantly. Then the patient was treated empirically with vancomycin and cefoperazone. The patient reported ongoing knee pain and fever (range from $37.1^{\circ} \mathrm{C}$ to $37.8^{\circ} \mathrm{C}$ ), but no effusion or sinus. Despite the combination of these two antibiotics, his ESR and CRP remained elevated, respectively.

On the 12th day after the surgery, laboratory results were as follows: ESR of $100 \mathrm{~mm} /$ hour (normal range, 0 $15 \mathrm{~mm} /$ hour), CRP of $139 \mathrm{mg} / 1$ (normal, $<8.0 \mathrm{mg} / \mathrm{l}$ ), hemameba of $10.9 \times 10^{9} / \mathrm{L}$ (normal range, 3.5-9.5 $\times 10^{9} / \mathrm{L}$ ), neutrophil of $9.12 \times 10^{9} / \mathrm{L}$ (normal range, $1.8-6.3 \times 10^{9} / \mathrm{L}$ ). Physical examination revealed a small effusion and a draining sinus over the anterior portion of the tibia on the left knee. Aerobic and anaerobic cultures of the synovial fluid were negative. Two days later, we performed the first phase of the debridement surgery in which synovium was removed and the polyethylene insert was replaced. During the operation, complete debridement was achieved through repeated irrigation of hydrogen peroxide, iodophor and saline solution. Meanwhile, we placed vancomycin into the knee joint and inserted drainage tubes to drain fluid. The drainage tube was pulled out 48 hours after surgery. Purulent material and inflammatory tissue were observed during surgery and three tissue specimens were submitted for aerobic and anaerobic culture. But bacterial, mycobacterial, and fungal culture were negative. The patient was treated empirically with vancomycin, cefoperazone and rifampicin.

To our surprise, after the debridement surgery, the patient still reported ongoing knee pain and fever (range from $37.4^{\circ} \mathrm{C}$ to $38.0^{\circ} \mathrm{C}$ ). The results of blood test suggested that ESR and CRP remained markedly elevated to $130 \mathrm{~mm} /$ hour and $239 \mathrm{mg} / \mathrm{l}$, respectively (Figure 1). Five days after the debridement surgery, he developed swelling and eventually drainage from two sinus tracts around the left knee and one sinus tract around the right knee. In view of the patient's clinical presentation and negative bacterial culture, metagenomic sequencing was used to identify the pathogen of the patient. The first metagenomic sequencing was carried on BioelectronSeq4000 instrument (Boao Institute of Biotechnology, Beijing, China) and this came back positive for Mycoplasma hominis. A second gene sequence analysis was also strongly positive for Mycoplasma hominis. Therefore, Mycoplasma hominis infection was confirmed. We then treated with a combination of moxifloxacin, doxycycline and imipenem. After about 15 days, the secretion was gradually decreased with the wound scabbed and cured in the end. The ESR, CRP, and temperature were all normal. Then, the patient took moxifloxacin and doxycycline orally for 6 months. At present, the patient has stopped antibiotics for one month, and has no knee pain and normal temperature.

\section{Discussion}

PJI can be caused by a wide range of pathogens, including gram-positive and gram-negative bacteria, anaerobic bacteria, and fungi. ${ }^{3,4,7}$ Staphylococcus proves to be the most common bacteria, accounting for about $70.0-80.0 \%$ of all types. ${ }^{8}$ However, in rare cases, PJI is caused by mycoplasma, especially when standard culture is negative. Identifying these pathogens by culture can be challenging for a variety of reasons, including prior antibiotic use, fastidious nature of the organisms, or growing in a biofilm state. ${ }^{8}$ This has significant implications for treatment because of the need to identify sensitive antibiotics.

Metagenomic sequencing is particularly useful in expediting the identification of slow-growing bacteria that may require several weeks of culture or display relevant biochemical reactions for identification purposes. ${ }^{9}$ Some case reports have demonstrated its value in the clinical setting of hard-todiagnose infections, particularly mycoplasma infections. ${ }^{8,10}$ Total nucleic acid was extracted from the sample. Both DNA and RNA sequencing libraries were constructed. Qualified DNA and RNA libraries were then amplified, quantitated and single-end sequenced on BioelectronSeq4000 sequencers. High quality data were obtained by removing low quality data and data with a length of less than $35 \mathrm{bp}$. Then, the data conforming to the human reference genome sequence were removed from the high quality data using the BurrowsWheeler Alignment. ${ }^{11}$ The remaining data were compared with four microbial databases including dedicated bacteria 

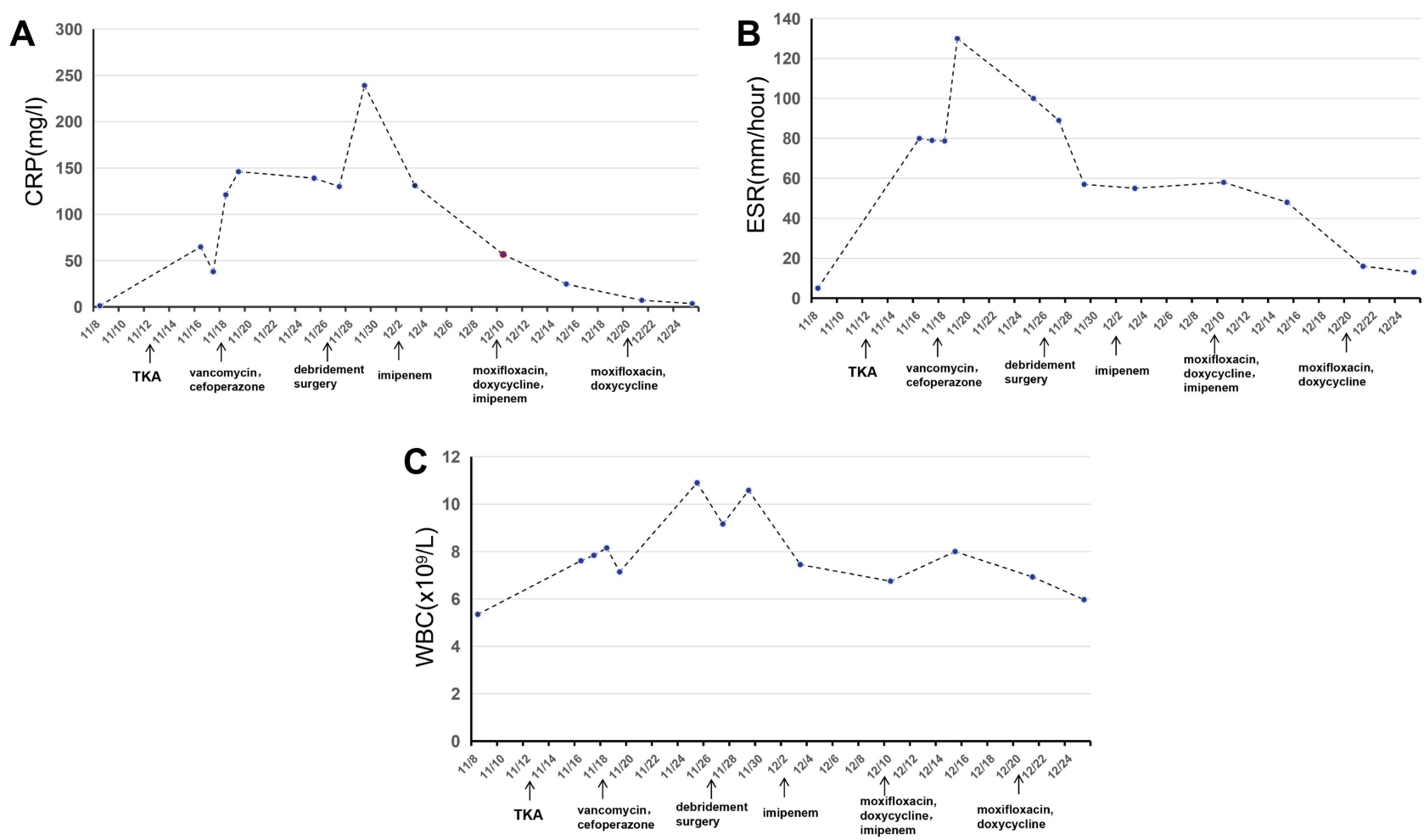

Figure I Changes of inflammatory markers during antibiotics treatment ((A) CRP, (B) ESR and (C) WBC).

(13,362 species), fungi (1659 species), viruses (7374 species), and parasites (153 species) to obtain the sequence number that could be matched with a certain pathogen. In this case, Mycoplasma hominis with 1251 readers was detected. The total length of this species genome was $688435 \mathrm{bp}$ and the total length of the measured species sequence splice was $113457 \mathrm{bp}$, with a coverage rate of $16.48 \%$.

Vancomycin and beta-lactam antibiotics are completely invalid for Mycoplasma hominis because their target is the cell wall. So our initial therapy including vancomycin and cefoperazone showed no efficacy against Mycoplasma hominis. The most common antimycoplasma drugs are divided into three groups: tetracycline, macrolides and fluoroquinolones. Owing to mutations in the 23S rRNA gene, Mycoplasma hominis is intrinsically resistant to C14 (roxithromycin and clarithromycin) and C15 macrolides (erythromycin and azithromycin). ${ }^{12}$ So we did not choose macrolides for treatment. Doxycycline was proved the most active choice for the treatment of Mycoplasma hominis. ${ }^{13,14}$ Quinolones or tetracyclines were active against Mycoplasma hominis and moxifloxacin appeared to have the greatest activity as the most effective therapeutic agent. ${ }^{15}$ The patient in our case therefore switched to the right regimen of the combination of moxifloxacin and doxycycline with a marked improvement of clinical syndromes and a favorable result. We also observed that the CRP level was relevant to the development of disease, while other indicators of infection did not change much.

In the current study, the number of reads uniquely aligned to the reference genome of Mycoplasma hominis was not very high. This may be due to the small amount of nucleic acid extracted from the specimen.

In conclusion, Mycoplasma hominis PJI should be considered in patients with culture-negative infection who do not respond to traditional antibiotics. CRP level is closely related to disease progression and is indicative of the entire course of treatment. The success of diagnosis in the present case indicates that metagenomic sequencing has great potential for diagnosing Mycoplasma hominis which provides strong support to guide physicians in selecting the appropriate pharmacotherapy.

\section{Ethics Approval}

Informed consent for our study was obtained from the patient.

\section{Funding}

There is no funding source. 


\section{Disclosure}

The authors declare that they have no competing interests.

\section{References}

1. Brockman BS, Maupin JJ, Thompson SF, et al. Complication rates in total knee arthroplasty performed for osteoarthritis and post-traumatic arthritis: a Comparison Study. J Arthroplasty. 2020;35(2):371-374. doi:10.1016/j.arth.2019.09.022

2. Klinder A, Zaatreh S, Ellenrieder M, et al. Antibiotics release from cement spacers used for two-stage treatment of implant-associated infections after total joint arthroplasty. J Biomed Mater Res B Appl Biomater. 2019;107(5):1587-1597. doi:10.1002/jbm.b.34251

3. Petis SM, Perry KI, Mabry TM, et al. Two-stage exchange protocol for periprosthetic joint infection following total knee arthroplasty in 245 knees without prior treatment for infection. $J$ Bone Joint Surg Am. 2019;101(3):239-249. doi:10.2106/JBJS.18.00356

4. Kurtz SM, Lau E, Schmier J, et al. Infection burden for hip and knee arthroplasty in the United States. J Arthroplasty. 2008;23(7):984-991. doi:10.1016/j.arth.2007.10.017

5. Valentine-King MA, Brown MB. Antibacterial resistance in Ureaplasma species and MYCOPLASMA hominis isolates from urine cultures in college-aged females. Antimicrob Agents Chemother. 2017;61(10):e01104-e01117. doi:10.1128/ AAC.01104-17

6. Moridi K, Hemmaty M, Azimian A, et al. Epidemiology of genital infections caused by Mycoplasma hominis, M. genitalium and Ureaplasma urealyticum in Iran; a systematic review and metaanalysis study (2000-2019). BMC Public Health. 2020;20:1020.

7. Springer BD, Scuderi GR. Evaluation and management of the infected total knee arthroplasty. Instr Course Lect. 2013;62:349-361.
8. Thoendel M, Jeraldo P, Greenwood-Quaintance KE, et al. A novel prosthetic joint infection pathogen, Mycoplasma salivarium, Identified by metagenomic shotgun sequencing. Clin Infect Dis. 2017;65(2):332-335. doi:10.1093/cid/cix296

9. Woo PC, Lau SK, Teng JL, et al. Then and now: use of 16S rDNA gene sequencing for bacterial identification and discovery of novel bacteria in clinical microbiology laboratories. Clin Microbiol Infect. 2008;14:908-934.

10. Han Z, Burnham CA, Clohisy J, et al. Mycoplasma pneumoniae periprosthetic joint infection identified by $16 \mathrm{~S}$ ribosomal RNA gene amplification and sequencing: a case report. J Bone Joint Surg Am. 2011;93:e103. doi:10.2106/JBJS.K.00318

11. Li H, Durbin R. Fast and accurate short read alignment with Burrows-Wheeler transform. Bioinformatics. 2009;25 (14):1754-1760. doi:10.1093/bioinformatics/btp324

12. Kechagia N, Bersimis $\mathrm{S}$, Chatzipanagiotou $\mathrm{S}$, et al. Incidence and antimicrobial susceptibilities of genital mycoplasmas in outpatient women with clinical vaginitis in Athens, Greece. $J$ Antimicrob Chemother. 2008;62:122-125. doi:10.1093/jac/dkn158

13. Maraki S, Mavromanolaki VE, Nioti E, et al. Prevalence and antimicrobial susceptibility of Ureaplasma species and Mycoplasma hominis in Greek female outpatients, 2012-2016. J Chemother. 2018;30:140-144. doi:10.1080/1120009X.2017.1404287

14. Skiljevic D, Mirkov D, Vukicevic J. Prevalence and antibiotic susceptibility of Mycoplasma hominis and Ureaplasma urealyticum in genital samples collected over 6 years at a Serbian University hospital. Indian J Dermatol Venereol Leprol. 2016;82:37-41. doi:10.4103/0378-6323.172903

15. Xiang L, Lu B. Infection due to Mycoplasma hominis after left hip replacement: case report and literature review. BMC Infect Dis. 2019;19(1):50. doi:10.1186/s12879-019-3686-z
International Journal of General Medicine

\section{Publish your work in this journal}

The International Journal of General Medicine is an international, peer-reviewed open-access journal that focuses on general and internal medicine, pathogenesis, epidemiology, diagnosis, monitoring and treatment protocols. The journal is characterized by the rapid reporting of reviews, original research and clinical studies across all disease areas. The manuscript management system is completely online and includes a very quick and fair peer-review system, which is all easy to use. Visit http://www.dovepress.com/ testimonials.php to read real quotes from published authors. 